Corresponding author: alyson.macinnes@philips.com

(C) 2019 Daniels et al. This article is distributed under the terms of the Creative Commons Attribution-NonCommercial License, which permits reuse and redistribution, except for commercial purposes, provided that the original author and source are credited.

Ontology terms: lethal infantile mitochondrial myopathy; refractory sideroblastic anemia

Published by Cold Spring Harbor Laboratory Press

doi:10.1101/mcs.a004457

\section{A uniparental isodisomy event introducing homozygous pathogenic variants drives a multisystem metabolic disorder}

\author{
Eileen G. Daniels, ${ }^{1}$ Marielle Alders ${ }^{2}$ Marco Lezzerini, ${ }^{1}$ Andrew McDonald, ${ }^{1}$ \\ Marjolein Peters, ${ }^{3}$ Taco W. Kuijpers, ${ }^{4}$ Phillis Lakeman, ${ }^{2}$ Riekelt H. Houtkooper, ${ }^{1}$ \\ and Alyson W. Maclnnes ${ }^{1,5}$

\begin{abstract}
${ }^{1}$ Laboratory of Genetic Metabolic Diseases, Amsterdam Gastroenterology and Metabolism, ${ }^{2}$ Department of Clinical Genetics, ${ }^{3}$ Department of Pediatric Hematology, ${ }^{4}$ Department of Pediatric Hematology, Immunology and Infectious Diseases, Emma Children's Hospital, Amsterdam UMC, University of Amsterdam, 1105 AZ Amsterdam, Netherlands
\end{abstract}

\begin{abstract}
Uniparental isodisomy (UPiD) is a rare genetic event that occurs when two identical copies of a single chromosome are inherited from one parent. Here we report a patient with a severe, multisystem metabolic disorder who inherited two copies of Chromosome 12 from her father. He was a heterozygous carrier of a variant in the muscle-specific enzyme 6-phosphofructokinase (PFKM) gene and of a truncating variant in the pseudouridine synthase 1 (PUS1) gene (both on Chromosome 12), resulting in a homozygous state of these mutations in his daughter. The PFKM gene functions in glycolysis and is linked to Tarui syndrome. The PUS1 gene functions in mitochondrial tRNA processing and is linked to myopathy, lactic acidosis, and sideroblastic anemia (MLASA). Analysis of human dermal fibroblasts, which do not express PFKM, revealed a loss of PUS1 mRNA and PUS1 protein only in the patient cells compared to healthy controls. The patient cells also revealed a reduction of the mitochondrial-encoded protein MTCO1, whereas levels of the nuclear-encoded SDHA remained unchanged, suggesting a specific impairment of mitochondrial translation. Further destabilization of these cells is suggested by the altered levels of BAX, BCL-2, and TP53 proteins, alterations that become augmented upon exposure of the cells to DNA damage. The results illustrate the efficacy of UPiD events to reveal rare pathogenic variants in human disease and demonstrate how these events can lead to cellular destabilization.
\end{abstract}

[Supplemental material is available for this article.]

\section{INTRODUCTION}

Uniparental disomy (UPD) is a rare event in genetic inheritance that results from two copies of a single chromosome being inherited from one parent (Robinson 2000; King et al. 2014). To date, approximately 3650 cases have been reported to involve all but four of the 22 autosomes (Liehr 2019). The consequences of UPD can be grave, potentially resulting in the homozygous inheritance of pathogenic autosomal recessive variants in case of uniparental isodisomy (UPiD) and/or abnormal genomic imprinting, which can happen both in UPiD and uniparental heterodisomy (UPhD). The resulting syndromic conditions depend largely

\footnotetext{
${ }^{5}$ Present address: Philips Research, Department of Precision Diagnostics, 5656 AE Eindhoven, Netherlands
} 
on the specific chromosome involved and include a wide range of clinical phenotypes including but not limited to neurodevelopmental disorders, kidney disease, growth irregularities, and metabolic disorders (Robinson 2000).

Here, we present a unique case of a female patient with a uniparental paternal isodisomy of Chromosome 12 (UPiD12pat), who presented with mild psychomotor developmental delay, microcephaly, short stature, hypotonia, exercise intolerance, myopia, and progressive transfusion-dependent sideroblastic anemia without major immunological defects. The UPiD12pat isodisomy resulted in homozygous variants in the muscle-specific enzyme 6-phosphofructokinase (PFKM) gene and the pseudouridine synthase 1 (PUS1) gene driving a multisystem metabolic disorder.

A multitude of metabolic disorders, which mostly have an autosomal recessive inheritance pattern, stem from variants that reduce the formation of ATP. This reduction can occur by impairing a number of different pathways. Enzyme deficiencies that reduce glycolysis and the breakdown of glucose can reduce downstream ATP formation-for example homozygous or compound heterozygous variants in the PFKM gene that are linked to glycogen storage disease VII, also called Tarui syndrome (GSD7; MIM \#232800). Variants that specifically impair mitochondrial protein translation can also lead to a loss of ATP through the reduced synthesis of proteins required for oxidative phosphorylation (OXPHOS) in the mitochondria. Variants in mitochondrial tRNAs are reported to drive defects in the central nervous system, muscle, and heart (Yarham et al. 2010) as are variants in nuclear genes coding for tRNA synthetases and mitochondrial ribosomal proteins (RPs) (Boczonadi and Horvath 2014).

Mitochondrial myopathy, lactic acidosis, and sideroblastic anemia (MLASA) is a rare autosomal recessive metabolic disorder that impairs OXPHOS and iron metabolism (Bykhovskaya et al. 2004). There are three subtypes of MLASA that are associated with specific variants in genes (found in either nuclear or mitochondrial DNA) coding for proteins that are important for mitochondrial translation and/or OXPHOS. MLASA1 (MIM \#600462) is associated with variants in the PUS1 gene (Bykhovskaya et al. 2004), which codes for an enzyme required for the pseudouridylation of nuclear and mitochondrial tRNAs (Patton et al. 2005). MLASA2 (MIM \#613561) is linked to variants in the YARS2 gene coding for a tyrosine tRNA synthetase enzyme (Riley et al. 2010). In contrast to variants in these nuclear genes involved in mitochondrial translation, MLASA3 (MIM \#500011) is linked to a variant in the MT-ATP6 gene, which is found on mitochondrial DNA and codes for a subunit of a large enzyme complex called ATP synthase (also known as complex V) that is important for OXPHOS (Burrage et al. 2014). Thus, these collective variants linked to MLASA can reduce OXPHOS by decreasing the levels of proteins required for complex formation or by impairing the mitochondrial translation machinery required for generating the proteins involved in complex formation.

Patients with MLASA typically present in late childhood with exercise intolerance, muscle weakness, and in some cases mild intellectual disability and dysmorphic features. Sideroblastic anemia is also often present, a condition defined by the accumulation of iron-laden mitochondria in a ring around the nucleus because of the inability of the cell to incorporate iron into hemoglobin (Inbal et al. 1995; Casas and Fischel-Ghodsian 2004; Zeharia et al. 2005; Fernandez-Vizarra et al. 2007; Metodiev et al. 2015; Cao et al. 2016; Kasapkara et al. 2017). This deficiency in iron metabolism is suggested to be the result of impaired mitochondrial translation in MLASA characterized by reduced OXPHOS complex activity and abundance (Richardson et al. 2010; Riley et al. 2010; Fleming 2011). Because iron sulfur cluster-containing proteins are involved in the electron transport system of the mitochondria, it is likely that the impaired complex formation disturbs the iron homeostasis contributing to the iron overload as observed in sideroblastic anemia (Richardson et al. 2010; Tesarova et al. 2019).

Defects that affect protein translation in the cytoplasm rather than in the mitochondria are also reported to drive anemia in humans. One example is Diamond-Blackfan anemia (DBA; 
MIM \#105650), an inherited bone marrow failure disorder and red cell aplasia that is linked to haploinsufficiency of several (20 to date) different RP genes, the most common of which is RPS19 (Ulirsch et al. 2018). One mechanism of action reported to drive the loss of hematopoietic progenitor cells in the bone marrow of DBA patients carrying RP gene variants is the stabilization of the TP53 tumor suppressor protein (Dutt et al. 2011). TP53 is normally kept at low levels in cells by binding to the E3-ubiquitin ligase MDM2 protein, the interaction resulting in the constitutive degradation of TP53 (Danilova et al. 2008). In the classical model of TP53 activation, cellular stress signals such as DNA damage or nucleolar stress disrupt the MDM2/ TP53 interaction, resulting in TP53 stabilization, translocation to the nucleus, and binding to target genes that when expressed either stop the cell cycle, such as p21, and initiate DNA repair or induce apoptosis depending on the level of damage (Vousden and Lane 2007).

Although the classical TP53 pathway focuses predominantly on the function of the stabilized protein as a transcription factor, it is now widely understood that a cytoplasmic pool of TP53 has an alternate method for promoting apoptosis involving the mitochondria. In this nonnuclear pathway TP53 translocates to the outer mitochondrial membrane in response to stress and interacts with the pro- and anti-apoptotic BCL-2 family members to activate or inhibit them (Vaseva and Moll 2009). For example, in response to DNA damage induced by UV, TP53 directly binds to the prosurvival BCL-2 protein on the outer mitochondrial membrane, displacing it from heterodimers formed with proapoptotic BAX and resulting in BCL-2 degradation. In the absence of BCL-2, BAX proteins are able to oligomerize with other BAX or Bak proteins and form channels in the mitochondrial membrane that allow the release of cytochrome $c$, the subsequent activation of the caspase cascade, and ultimately apoptosis (Zinkel et al. 2006). Thus, the ratio of BCL-2:BAX proteins has emerged as an important indicator as to whether a cell is being driven toward survival or apoptosis (Latonen et al. 2001; Raisova et al. 2001).

This study reports a patient with a multisystem metabolic disorder involving early-onset MLASA caused by an extremely rare UPiD event that resulted in the inheritance of homozygous pathogenic variants in genes reported to drive metabolic disease. The subsequent analysis on primary patient cells was performed in order to test how these variants affected cellular function focusing on mitochondrial translation and the downstream effects of its impairment in the context of the TP53 pathway.

\section{RESULTS}

\section{A Homozygous Variant c.1122C > G in PUS1 Identified in a Patient with MLASA}

Here, we report a young female patient who was referred to the clinic at $4 \mathrm{yr}$ of age. She presented with mild psychomotor developmental delay $(\mathrm{IQ} \sim 80)$, microcephaly $(<-2,5 \mathrm{SD})$, short stature ( $-3 \mathrm{SD})$, hypotonia, exercise intolerance, myopia, and progressive transfusion-dependent sideroblastic anemia without major immunological defects (see Supplemental Table S1 for complete blood count). She was the only child of nonconsanguineous Caucasian parents.

Clinical examination at first presentation showed mild facial dysmorphisms with upslanting palpebral fissures, hypertelorism, hypoplastic alae nasi, a smooth philtrum, small chin, and cup-shaped and low implanted ears. Furthermore, there was a tapering of the fingers, bilateral clinodactyly of the fifth digits, vitiligo, and mild eczema. Biochemical investigations revealed a slightly elevated lactate in the blood with no amino acid abnormalities (data not shown). At the age of $7 \mathrm{yr}$ and 4 mo the patient received a stem cell transplantation for her sideroblastic anemia and unexpectedly died because of a viral gastroenteritis with metabolic dysregulation. No permission for autopsy was gained and no muscle biopsy was performed during her life or after she passed away. 
Initial genetic testing showed no abnormalities in karyotyping $(46, \mathrm{XX})$ or in microarray $\mathrm{CGH}$ analysis. A mitomycin $\mathrm{C}$ test excluded Fanconi anemia (FA; MIM \#227650). The presence of myopathy and bone marrow failure suggested a possible diagnosis of Pearson syndrome; however, targeted sequencing of mtDNA excluded this possibility. DBA was also considered; however, targeted sequencing of RPS19 and RPS24 (commonly mutated genes in DBA [Campagnoli et al. 2008]) and at the time the only RP genes that were part of the targeted sequencing panel) revealed these genes to be wild type. Genes (SHOX, FGFR3, NBS1) linked to Nijmegen breakage syndrome (NBS; MIM \#251260) and several sideroblastic anemia (SIDBA1; MIM \#300751) genes (SLC25A38; GLR5, STEAP3, ABCB7, ALAS2) were also sequenced and found to be wild type.

Trio whole-exome sequencing (WES) was then performed after the targeted sequencing of the disease genes described above. This revealed homozygosity of several variants on Chromosome 12 of the patient (Fig. 1A; Supplemental Fig. S1). The father of the patient was found to be a heterozygous carrier of these variants, and the mother's DNA only revealed the wild type. The absence of heterozygous variants on Chromosome 12 suggested the occurrence of a paternal UPiD in which the patient inherited two identical copies of one Chromosome 12 from her father (Fig. 1A,B; Supplemental Fig. S1). Two of these variants were identified as potentially pathogenic: (1) a previously unreported homozygous variant in the PUS1 gene (c.1122C > G, p.Tyr374*; NM_025215.5) which is associated with mitochondrial myopathy, lactic acidosis, and sideroblastic anemia (MLASA1; MIM \#600462) and (2) a homozygous splice site variant in PFKM (c.237 + 1G > A, NM_000289.5), which is associated with GSD7, also called Tarui syndrome (Supplemental Fig. S1).

Sanger sequencing performed in patient fibroblasts confirmed the homozygous nonsense variant in PUS1 (c.1122C > G) predicted to replace the codon for a tyrosine at position 374 with a TAG stop codon (Fig. 1C) and the splice site variant in PFKM (Supplemental Fig. S2). Although the allele frequency for the PFKM variant (ClinVar [https://www.ncbi.nIm.nih .gov/clinvar] accession number VCV000189239) is calculated to be between 0.00006 and 0.00008 (Trans-Omics for Precision Medicine and Exome Aggregation Consortium, respectively), variants in the PUS1 gene are exceptionally rare. Only 15 individuals to date have been reported in the literature with PUS1 variants (excluding this study), including one individual who was erroneously diagnosed with DBA (Ulirsch et al. 2018) (see Supplemental Table S2 for full description of previously reported PUS1 variants). The new PUS1 variant information was submitted to ClinVar and has the accession number SCV000995055 (see Table 1 for variant details).

Quantitative PCR (qPCR) analysis of primary fibroblasts derived from the affected individual revealed a significant reduction in PUS1 mRNA levels compared to fibroblasts from a healthy control (Fig. 1D). This was confirmed by western blotting using an antibody recognizing both PUS1 protein isoforms in the healthy control but not in patient cells (Fig. 1E). Another primary antibody recognizing a different epitope of PUS1 also showed little remaining PUS1 protein in the same fibroblasts (Supplemental Fig. S3). We also measured the levels of PFKM mRNA in healthy control and patient fibroblasts by qPCR. We did not detect any PFKM mRNA in either cell line, confirming our expectation that PFKM expression is restricted to muscle (Raben and Sherman 1995) and should not be found in dermal fibroblasts (Supplemental Fig. S4).

Confocal microscopy analysis of healthy control fibroblasts with antibodies against PUS1 proteins and TOMM20 proteins (which are used as markers of the mitochondria) revealed the expected colocalization of PUS1 with the nucleus and mitochondria (shown previously with HA-tagged PUS1 constructs [Fernandez-Vizarra et al. 2007]). In contrast, negligible staining of PUS1 was detected in the patient fibroblasts, with a complete loss of nuclear PUS1 staining and no observable colocalization with TOMM20 and the mitochondria (Fig. 1F). This result is in line with the observation that there is little remaining 
A

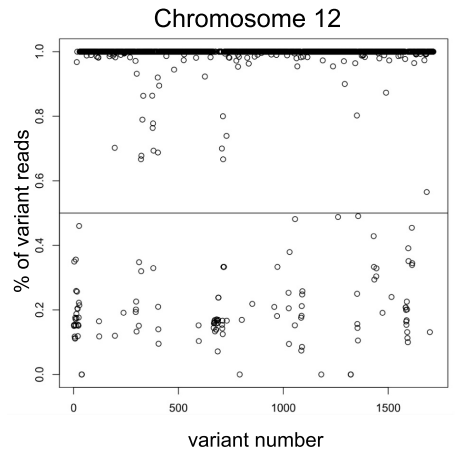

C

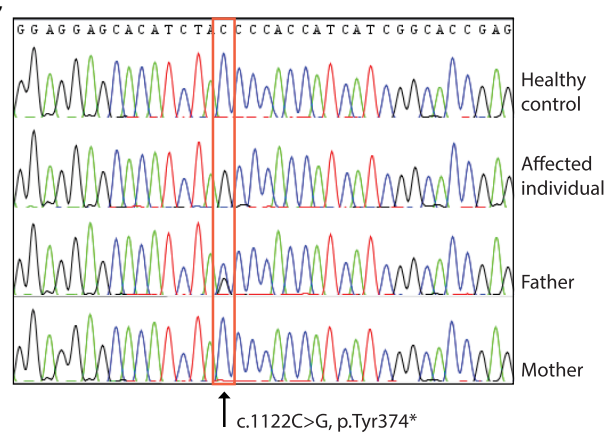

Chromosome 13

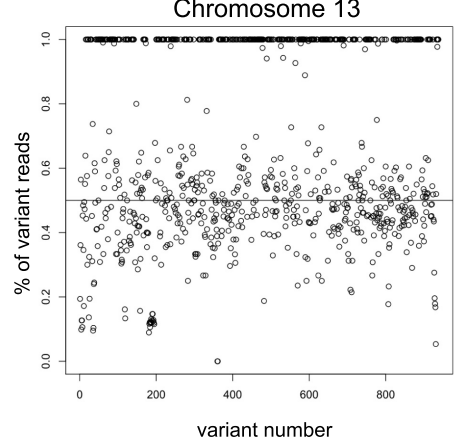

D

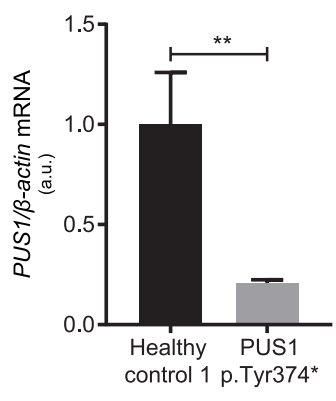

B

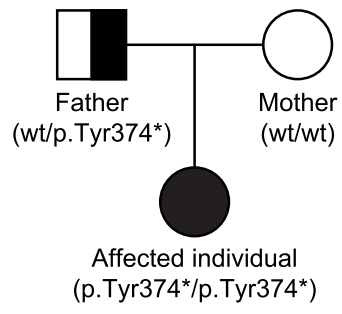

E

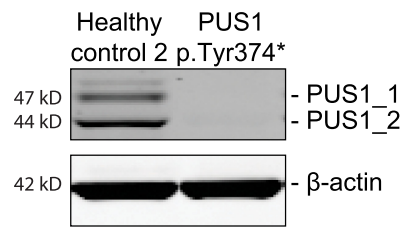

$\mathbf{F}$

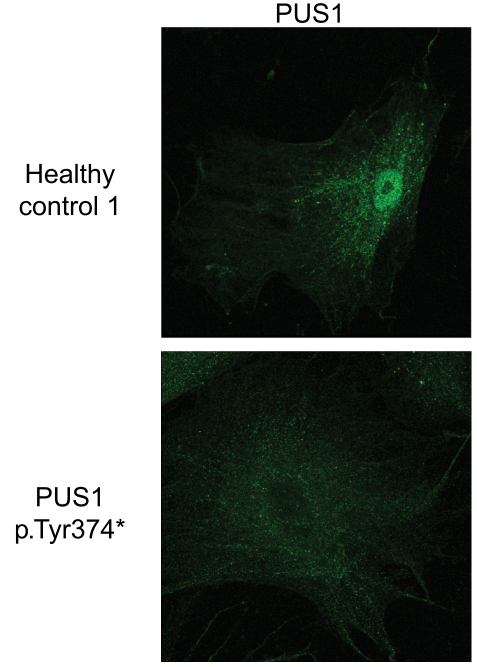

TOMM20
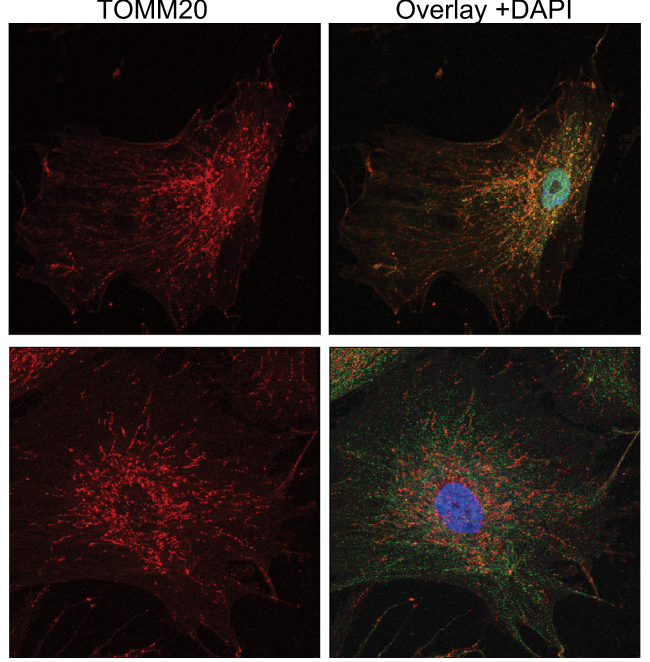

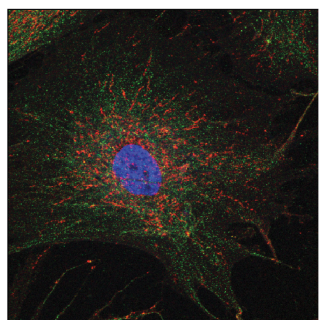

Figure 1. Identified homozygous variant c. $1122 \mathrm{C}>\mathrm{G}$ reduces PUS1 mRNA and protein levels. $(A)$ The percentage of variant reads for all variants on Chromosome 12 identified by whole-exome sequencing (left). This demonstrates an absence of heterozygous variants, which was not seen for other chromosomes-for example, Chromosome 13 (right). (B) Pedigree of the affected individual illustrating paternal isodisomy. (C) Sanger sequencing of fibroblasts from the affected individual illustrating the homozygous $C>G$ point mutation introducing a nonsense stop codon. (D) Quantitative polymerase chain reaction (qPCR) measuring mRNA expression of PUS1 in healthy control (black) and the affected individual (gray) in fibroblasts, normalized to $\beta$ actin mRNA. Data are represented as the mean $(N=3),\left({ }^{* *}\right) P<0.005$ as calculated by a two-tailed Student's ttest. (E) Western blot showing the reduced PUS1 protein levels in fibroblasts derived from the affected individual compared to a healthy control. All western blots shown are representative of at least three independent experiments. (F) Confocal microscopy of fibroblasts illustrating the localization of PUS1 (green), mitochondria demarked by TOMM20 (red), and an overlay (yellow) including DAPI (blue) staining. 
Table 1. Description of variants described in this study

\begin{tabular}{|c|c|c|c|c|c|c|c|c|}
\hline Gene & Chromosome & $\begin{array}{l}\text { HGVS DNA } \\
\text { reference }\end{array}$ & $\begin{array}{l}\text { HGVS protein } \\
\text { reference }\end{array}$ & Variant type & $\begin{array}{l}\text { Predicted } \\
\text { effect }\end{array}$ & $\begin{array}{c}\mathrm{dbSNP} / \mathrm{db} \text { Var } \\
\text { ID }\end{array}$ & Genotype & ClinVar ID \\
\hline PFKM & 12 & c. $237+1 G>A$ & N/A & $\begin{array}{l}\text { Single- } \\
\text { nucleotide } \\
\text { variant }\end{array}$ & Splice donor & rs202143236 & Homozygous & VCV000189239 \\
\hline PUS1 & 12 & c. $1122 C>G$ & p.Tyr374* & $\begin{array}{l}\text { Single- } \\
\text { nucleotide } \\
\text { variant }\end{array}$ & Nonsense & N/A & Homozygous & SCV000995055 \\
\hline
\end{tabular}

PUS1 mRNA and no detectable PUS1 protein in the primary fibroblasts derived from the affected individual.

\section{Absence of PUS1 Likely Impairs Mitochondrial Translation}

Because PUS1 is required for the proper pseudouridylation of tRNAs necessary for efficient mitochondrial translation, we assessed the protein levels of succinate dehydrogenase complex (SDHA) and cytochrome c oxidase subunit I (MTCO1). Although both SDHA and MTCO1 proteins are localized to mitochondria and are required subunits for the formation of OXPHOS complexes, only MTCO1 is encoded by a mitochondrial DNA gene. We observed a significant reduction in MTCO1 expression in the fibroblasts carrying the PUS1 variant, whereas the SDHA levels in these cells remained equal to the levels in healthy control cells (Fig. 2A,B). These results suggest that in line with previous reports noted above (Fernandez-Vizarra et al. 2007), the loss of PUS1 protein specifically drives a reduction of mitochondrial protein translation.

\section{Fibroblasts Carrying the PUS1 Variant Reveal Destabilization and Hypersensitivity to UVC Exposure}

Because defects in the cytoplasmic translation are widely reported to activate the TP53 pathway (Dutt et al. 2011), we measured TP53 stabilization in fibroblasts carrying the PUS1 c. $1122 \mathrm{C}>\mathrm{G}$ variant in order to determine if impaired mitochondrial translation also affects
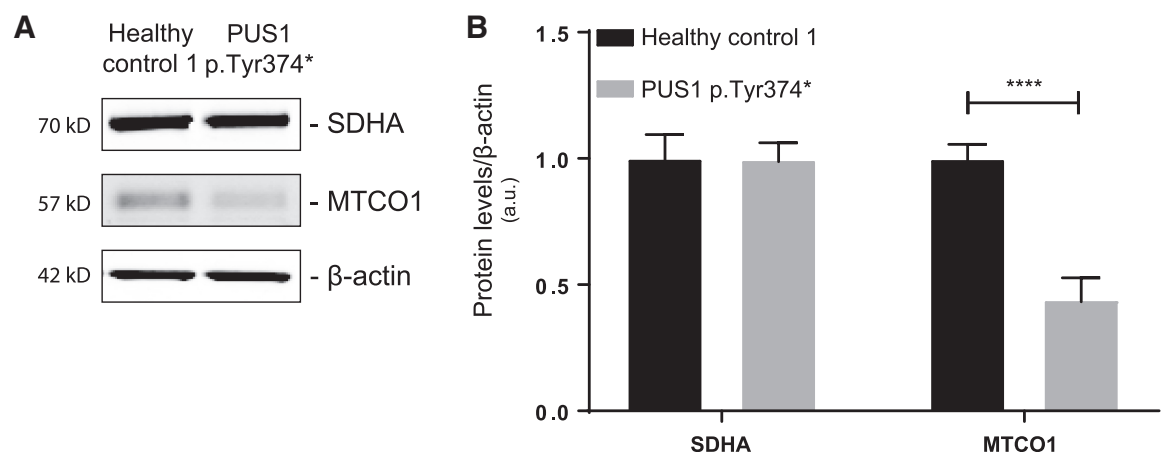

Figure 2. The PUS1 variant specifically impairs mitochondrial translation. (A) Western blot showing the protein levels of the nuclear-encoded SDHA compared to the mitochondrial-encoded MTCO1 in fibroblasts carrying the PUS1 p.Tyr374* variant or derived from a healthy control. (B) Quantification of the results in $A$. The levels of SDHA (left) and MTCO1 (right) in fibroblasts from the affected individual (gray) and healthy control (black) normalized to $\beta$-actin. Protein levels of MTCO1 are significantly reduced in cells from affected individual compared to healthy control, whereas the SDHA protein levels remain stable. Data are represented as the mean $(N=3),\left(*^{* * *}\right) P<0.00005$ as calculated by a two-tailed Student's t-test. 
the pathway. For these experiments PUS1 variant and healthy control fibroblasts were untreated or exposed to $50 \mathrm{~J} / \mathrm{m}^{2}$ UVC radiation. Twenty-four hours after exposure cells were collected for analysis of the levels of proteins involved in the TP53 activation pathway

(Fig. 3A). The levels of TP53 were significantly increased in patient fibroblasts compared

A

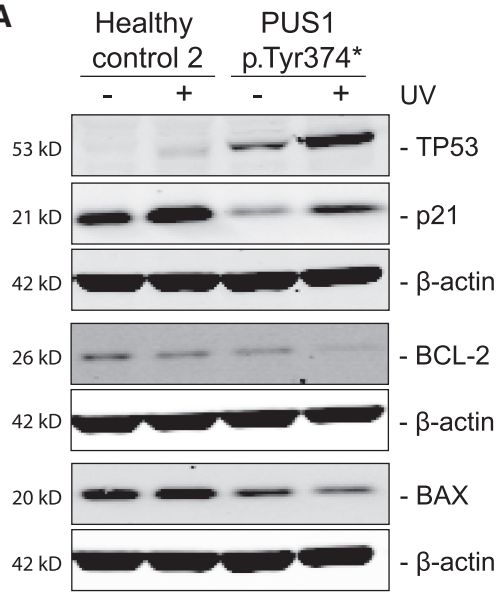

B

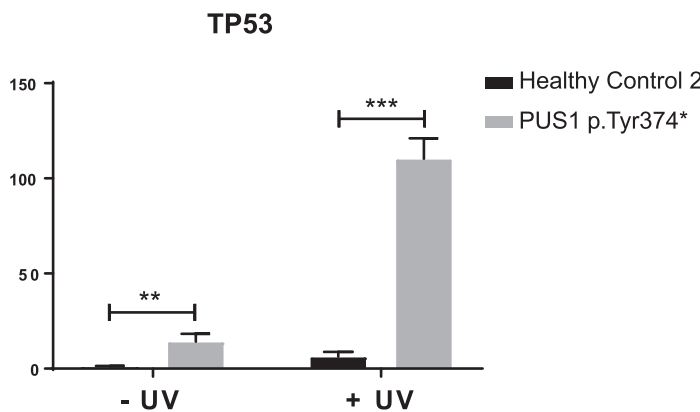

D

BCL-2 p21
- Healthy Control 2 PUS1 p.Tyr374*

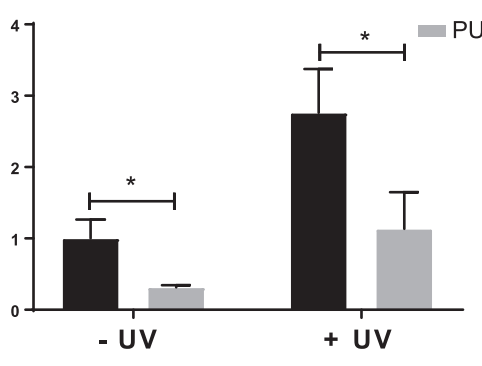

BAX

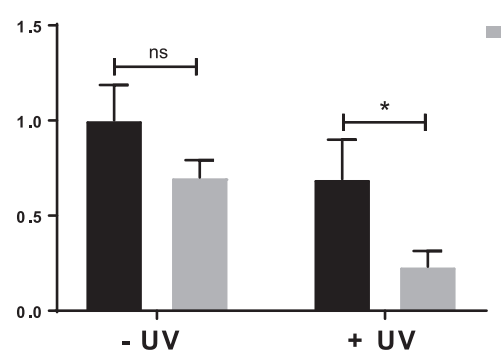

$\mathbf{F}$
E

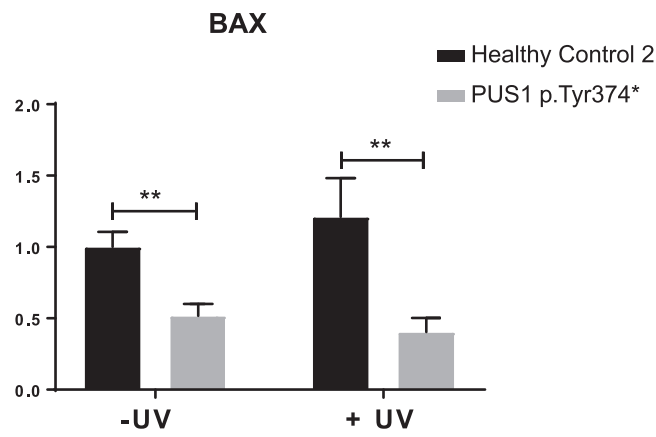

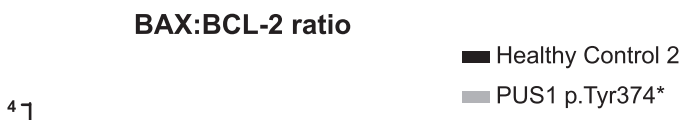

Figure 3. Cells carrying the PUS1 p.Tyr374* variant stabilize TP53 and alter mitochondrial protein BCL-2 and BAX levels. (A) Western blot showing the protein levels of TP53, p21, BCL-2, and BAX in fibroblasts derived from a healthy control or the affected individual upon $50 \mathrm{~J} / \mathrm{m}^{2} \mathrm{UVC}$ exposure after $24 \mathrm{~h}$. (B) Protein levels of TP53 were significantly increased in cells from affected individual (gray) under basal conditions (-UV) and further increased upon UVC exposure (+UV) compared to control cells (black). (C) Protein levels of p21 were significantly lower in cells from the affected individual under both conditions; however, p21 was still induced upon UVC exposure. (D) BCL-2 and (E) BAX protein levels were reduced in cells from the affected individual under both conditions. $(F)$ The ratio of $\mathrm{BAX}: \mathrm{BCL}-2$ is shown to remain stable in cells from affected individual and control cells under both conditions. Data are represented as the mean $(N=3),(*) P<0.05,\left({ }^{* *}\right) P<0.005,\left({ }^{* * *}\right) P<$ 0.0005 as calculated by a two-tailed Student's t-test. 
to healthy control cells under basal conditions (Fig. 3A,B). Moreover, exposing cells to UVC revealed a much stronger induction of TP53 stabilization compared to what was observed in healthy control cells exposed to UVC (Fig. 3A,B).

The cyclin-dependent kinase inhibitor p21 (WAF1/CDKN1A) is a nuclear gene and direct target of TP53 that is up-regulated in response to several kinds of cellular stress (el-Deiry et al. 1993). Despite the increased levels of TP53 under basal conditions in the patient fibroblasts compared to healthy control cells, levels of 21 in the same conditions were markedly reduced in the patient cells (Fig. 3A,C). In both patient and healthy control cells a relatively similar induction of p21 protein is observed as a result of UVC exposure, suggesting that the TP53/p21 pathway remains unimpaired in the mutant cells (Fig. 3A,C). These results suggest that although an increase in TP53 stabilization is observed in cells carrying the PUS1 variant, this pool of TP53 is not targeting and up-regulating the classical p21 gene target in the nucleus.

We next measured levels of the outer mitochondrial membrane proteins BCL-2 and BAX. The levels of both these proteins were reduced in the cells carrying the PUS1 variant, both with or without UVC (Fig. 3A,D,E). Interestingly, the concomitant decrease of BCL-2 and BAX results in a relatively unchanged BAX:BCL-2 ratio in the cells carrying the PUS1 variant compared to healthy control cells (Fig. 3F), whereas an increase of this ratio would have been suggestive of apoptosis (Zinkel et al. 2006; Zhou and Chang 2008). Taken all together, these results suggest that although cells carrying the PUS1 variant stabilize TP53 under basal conditions, this does not result in the up-regulation of target genes such as p21. However, the degradation of BCL-2 in the mutant cells under basal conditions suggests destabilization of the mitochondria. The concomitant reduction of BAX suggests that the cells carrying the PUS1 variant are able to equilibrate the BAX:BCL-2 ratio by reducing BAX levels in the outer mitochondrial membrane, perhaps as a mechanism to prevent cytochrome $c$ release from the mitochondria and evade apoptosis. This hypothesis is supported by electron micrographs of healthy control and patient fibroblasts showing that stress-induced changes in mitochondria, such as their fragmentation and disorganization/swelling of their cristae (Perkins et al. 2009), are only evident in the PUS1 mutant cells $24 \mathrm{~h}$ after UVC exposure, and no major changes are evident in cells at basal levels (Fig. 4).

\section{DISCUSSION}

This study describes a young female child who was diagnosed with mitochondrial myopathy, lactic acidosis, and sideroblastic anemia type 1 (MLASA1) and with glycogen storage disease VII (GSD7) resulting from a paternal uniparental isodisomy of Chromosome 12 (UPiD12pat) event. A UPD is a rare event and it usually arises as a result of rescue of a trisomy or monosomy in a zygote (Robinson 2000; King et al. 2014). In the case of a trisomy rescue, one of the three chromosomes is lost, and when the two remaining chromosomes originate from the same parent, the result is a UPD. As recombination occurs during meiosis, these chromosomes have parts of heterodisomy (two different copies of the same parent) and parts of isodisomy (same copies of the same parent) (Balbeur et al. 2016). Because the patient carried two completely identical copies of the paternal Chromosome 12, we suspect that the mode of UPD formation in this case was the rescue of a monosomy, by duplication of the single paternal chromosome in the zygote, resulting in a complete UPiD.

The UPiD12pat resulted in inheritance of homozygous pathogenic variants: one previously reported variant (c.237 +1G $>$ A) in PFKM that impairs glycolysis and an unreported early stop codon (c.1122C > G; p.Tyr374*) in the PUS1 gene. The identification of this latter homozygous variant makes this the 16th case known with MLASA1 linked to PUS1 (see Supplemental Table S2). Primary fibroblasts showed decreased PUS1 mRNA expression and protein levels compared to healthy control cells, suggesting the mRNA is subject to 
COLD SPRING HARBOR Molecular Case Studies
Homozygous variants and UPD

A

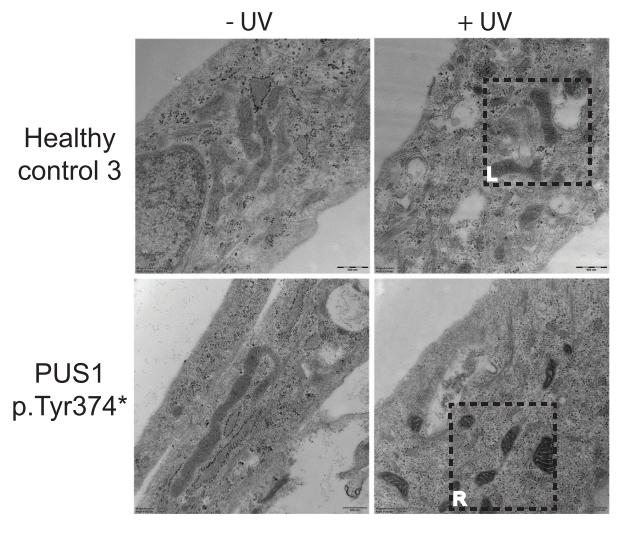

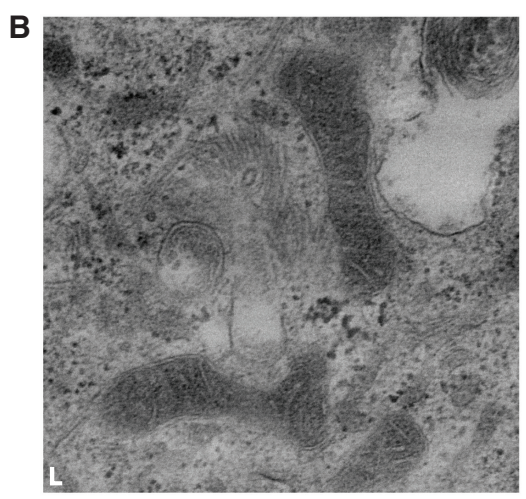

Healthy control 3

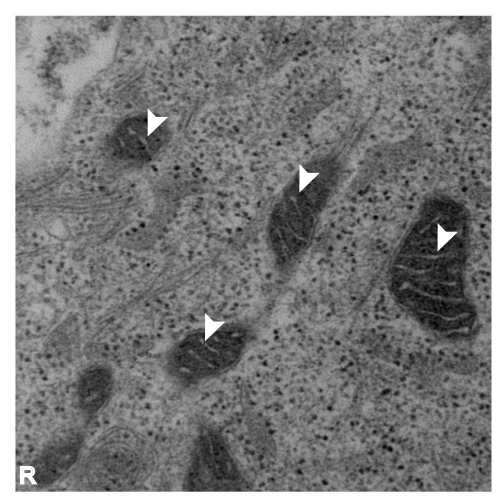

PUS1 p.Tyr374*

Figure 4. Electron microscopy of fibroblasts with and without UVC exposure. (A) Patient (PUS1 p.Tyr374*) (lower panels) and healthy control (upper panels) fibroblasts show no morphological difference at basal level (left panels). Twenty-four hours after exposure to $50 \mathrm{~J} / \mathrm{m}^{2} \mathrm{UVC}$ (+UV, right panels), note the increased fragmentation of mitochondria in the cells carrying the PUS1 variant in the dashed box outline labeled R. (B) Enlarged images of UVC-treated fibroblasts from a healthy control on the left $(\mathrm{L})$ and carrying the PUS1 p.374Tyr* variant on the right (R). White arrowheads indicate swollen cristae.

nonsense-mediated decay (Fig. 1D-F). Cells carrying the variant and depleted PUS1 protein levels revealed reduced levels of a specific mitochondrial-encoded protein, whereas levels of a nuclear-encoded protein remained unchanged, strongly suggesting that the variant is impairing mitochondrial translation (Fig. 2). In addition, primary patient cells revealed altered levels of BAX, BCL-2, and TP53 proteins suggestive of destabilization, especially upon exposure to UV (Fig. 3). The mitochondria in the patient cells also reveal a more abnormal and fragmented morphology compared to healthy control cells upon UV exposure (Fig. 4).

In contrast to the formerly described individuals who mostly presented with MLASA1 in late childhood (Supplemental Table S2), this patient presented at an early childhood age with a typical MLASA1 phenotype (e.g., exercise intolerance, lactic acidosis, and sideroblastic anemia), which are all reported features in patients with PUS1 variants (Inbal et al. 1995; Casas and Fischel-Ghodsian 2004; Zeharia et al. 2005; Fernandez-Vizarra et al. 2007; Metodiev et al. 2015; Cao et al. 2016; Kasapkara et al. 2017). However, it is highly likely that her clinical phenotype, such as the exercise intolerance and elevated lactate, was exacerbated by the second inherited homozygous variant (c.237 + 1G > A) in the splice site of the PFKM. In fact, this particular variant has been reported with some frequency in patients with GSD7. This disease often but not always results in exercise intolerance, myopathy, and red cell hemolysis (Raben and Sherman 1995). In fact, it is suspected that the clustering of 
reported variants linked to GSD7 disease may reflect a referral bias because individuals carrying pathogenic variants often have such mild clinical phenotypes that they do not seek medical treatment (Raben and Sherman 1995). Nonetheless, with the individual reported in this study we cannot attribute the clinical phenotype entirely to the PUS1 variant, and it seems very likely that both variants contributed to the severity of her condition. However, for the molecular biology studies in patient fibroblasts, the expression of PFKM (being a muscle-specific gene [Vora 1983]) in both healthy control and patient cells is negligible, which allows us to focus more exclusively on the downstream effects of PUS1 variant.

The reductions of the mitochondrial BCL-2 and BAX proteins in the cells carrying the PUS1 variant strongly suggest destabilization of the mitochondria, in line with the observation that the variant likely affects mitochondrial but not cytoplasmic translation (Fig. 2). Although it is known that PUS1 can function both in the cytoplasm and mitochondria, it is possible that the redundancy of the other PUS enzymes (PUS2-7) is able to compensate for the loss of PUS1 in the cytoplasm but not the mitochondria (Patton et al. 2005; Suzuki et al. 2011). However, we cannot rule out the possibility that the loss of PUS1 impairs cytoplasmic translation subtly in a way that is undetectable with our experimental methods.

The unchanged ratio between the reduced BCL-2 and BAX proteins in the cells carrying the PUS1 variant compared to healthy control cells is also an interesting observation that we cannot fully explain (Fig. 3F). Reductions of BCL-2 alone in response to cellular stress would drive the formation of channels in the outer mitochondrial membrane and the release of cytochrome $c$, which would then go on to initiate caspase cleavage and apoptosis (Zinkel et al. 2006; Zhou and Chang 2008; Fulda et al. 2010). Thus, like the reduction of p21, perhaps the cells carrying the PUS1 variant have devised a mechanism to evade apoptosis by concomitantly reducing levels of $\mathrm{BAX}$ and maintaining an equal $\mathrm{BAX}: \mathrm{BCL}-2$ ratio.

TP53 stabilization is known to be linked to syndromes with impaired cytoplasmic translation such as DBA, and cell-based studies show that variants driving this increase of TP53 also result in an increase of p21 at basal levels (Badhai et al. 2009; Pereboeva et al. 2013). In contrast, the fibroblasts used for the current study revealed a reduction of p21 in cells carrying the PUS1 variant compared to healthy control cells, although both healthy control and PUS1 mutant cell lines revealed the expected increase of p21 levels upon exposure to UVC (Fig. $3 A, C)$. These results suggest that the classical TP53 pathway response to DNA damage remains undisturbed in cells carrying the PUS1 variant, and also suggest that p21 levels are kept at low levels perhaps as a way for the cell cycle to proceed unimpeded by a mechanism that remains unknown. However, it is important for us to note here that we cannot be entirely sure as to what specifically is driving the TP53 stabilization in the patient cells. Because there are previous reports linking inherited mutations that drive mitochondrial disease to an increased susceptibility of cells to apoptosis (Liu et al. 2004), it seems likely that this augmented TP53 stabilization is due to the PUS1 variant. That said, we cannot rule out that this is an effect of general genomic instability driven by the UPiD or to some other unknown variable. Moreover, it remains unclear what the downstream clinical impact is resulting from the observed increase in TP53 stabilization in the fibroblasts. Although similar augmented TP53 stabilization has been observed-in bone marrow failures, syndromes linked to variants that impair cytoplasmic translation (such as DBA)—the pathophysiology explaining these genotype:phenotype correlations remains to be fully solved.

In conclusion, this study reports a rare UPiD event driving a complex multisystem metabolic disorder that resulted from the paternal inheritance of two homozygous pathogenic variants, one previously reported in PFKM and one unreported truncating variant in PUS1. The effects of these two variants on glycolysis and mitochondrial translation likely are responsible for driving the severity of the clinical phenotype. Moreover, primary cells from the patient suggest that there may be a link between impaired mitochondrial translation and the TP53 pathway, an area that will be very interesting for future research. 


\section{METHODS}

\section{Cell Culture}

Primary skin fibroblasts from patients and healthy controls were obtained from a punch biopsy on the upper arm and cultured on DMEM (Bio-Whittaker) medium supplemented with 10\% fetal bovine serum (Bio-Whittaker), $25 \mathrm{mM}$ HEPES buffer (Bio-Whittaker), $100 \mathrm{U} / \mathrm{mL}$ penicillin, $100 \mu \mathrm{g} / \mathrm{mL}$ streptomycin (Life Technologies), and $250 \mathrm{ng} / \mathrm{mL}$ Fungizone (Life Technologies). Cells were cultured at $37^{\circ} \mathrm{C}$ with $5 \% \mathrm{CO}_{2}$.

\section{Whole-Exome Sequencing}

WES was performed by the molecular DNA genetic technicians of the Department of Clinical Genetics of the Amsterdam UMC, Amsterdam. Whole-exome capture was performed using SeqCap EZ Human Exome Library v3.0 (Roche NimbleGen). The resulting enriched libraries were sequenced on a HiSeq 2500 platform (Illumina) according to the manufacturer's recommendations for paired-end 101-bp reads. A total of 44,308,329 reads were obtained from the proband 41,606,886 from the father and 37,140,533 from the mother. Alignment of sequence reads to human reference genome (hg19) was done using BWAMEM (bio-bwa.sourceforge.net/), and variants were called using the GATK3.2 software package (www .broadinstitute.org/gatk/). Mean coverage was 70 -fold for the proband, and $86 \%$ of the exome was covered with more than 20 reads. Filtering of variants was done using Cartagenia Bench Lab NGS (Agilent). Variants with fewer than five reads, a frequency of more than $1 \%$ in public (ESP, dbSNP, 1KG) and/or in-house databases were excluded. De novo, homozygous, or compound heterozygous variants present in exons or within $\pm 6 \mathrm{nt}$ in the intron were evaluated. This revealed homozygosity for the variant c.1122C $>$ G, p.Tyr374* in PUS1 (NM_025215.5) and variant c.237 + 1G > A in PFKM (NM_000289.5). Coverage at these variants was 110 -fold and 54 -fold, respectively. Both variants were confirmed by Sanger sequencing. Explicit permission for making the exome sequencing data publicly available was not granted.

\section{Sanger Sequencing}

Confirmation of the variants found by WES was done using Sanger sequencing. Primers were developed to amplify exon 5 of PUS1 (primer sequences available on request). After PCR products were sequenced using Bigdye v1.1 chemistry and an ABI3100 sequencer (Life Technologies). Sequences were analyzed using CodonCode Aligner (CodonCode Corporation).

\section{qPCR}

TRlzol Reagent (Sigma-Aldrich) was used to isolate RNA from fibroblasts. Genomic DNA was eliminated, and cDNA was made by the use of QuantiTect Reverse Transcription Kit (QIAGEN). qPCR samples were prepared with SYBR green (Roche), and qPCR was performed on the LightCycler 480 device (Roche).

The primers were PUS1 (Fw: CATGCAGAGGAATGTCGGGT, Rev: AATCAGCCACAC CTTCAGGG) and $\beta$-actin (Fw: AGTCATTCCAAATATGAGATGCGTT, Rev: GCTATCACCT CCCCTGTGTG).

\section{Western Blotting}

Protein was isolated from fibroblasts with the use of RIPA lysis buffer $(50 \mathrm{mM}$ Tris- $\mathrm{HCl}(\mathrm{pH}$ 7.4), $150 \mathrm{mM} \mathrm{NaCl}, 0.1 \%$ SDS, $0.5 \%$ sodium deoxycholate, $1 \%$ Triton $\mathrm{X}-100$ ) supplemented with Complete mini protease inhibitor (Roche). Subsequently, samples were sonicated to 
ensure complete lysis. The protein concentrations were determined using the BCA Protein Assay (Thermo Scientific). Protein samples were prepared with LDS sample buffer (NuPage, Invitrogen) and sample reducing agent (NuPage, Invitrogen). Protein samples were loaded onto a 4\%-12\% Tris-Bis Protein Gel (NuPage, Invitrogen). Gels were transferred to a nitrocellulose membrane using the iBlot Dry Blotting System. Membranes were blocked with $3 \%$ milk in PBS-T $\left(0.1 \%\right.$ Tween-20) and incubated with primary $\left(\mathrm{O} / \mathrm{N}, 4^{\circ} \mathrm{C}\right)$ and secondary $(1 \mathrm{~h}, \mathrm{RT})$ antibodies.

The primary antibodies were PUS1 (NBP1-32902, Novus Biologicals), PUS1 (sc-376443, Santa Cruz), MTCO1 (ab14705, Abcam), SDHA (ab14715, Abcam), TP53 (sc-6243, Santa Cruz), BCL-2 (ab692-500, Abcam), BAX (\#2772, Cell Signaling), and $\beta$-actin (A5441, Sigma-Aldrich).

The secondary antibodies were IRDye $800 \mathrm{CW}$ goat anti-rabbit (925-32211, LI-COR), IRDye 800CW goat anti-mouse (925-32210, LI-COR), and IRDye 680RD donkey anti-mouse (925-68072, LI-COR).

\section{Confocal Microscopy}

Cells were fixed with 2\% PFA-PBS, permeabilized with $0.1 \%$ triton-PBS, and blocked with $1 \%$ BSA-PBS buffer. Primary antibody was prepared in 1\% BSA-PBS (1:100) and secondary in 1\% PBS (1:200). Cells were washed and mounted with Prolong Gold antifade reagent with DAPI (Invitrogen p36935). The primary antibodies were PUS1 (sc-376443, Santa Cruz), and TOMM20 (ab70699, Abcam). The secondary antibodies were rabbit biotin (E0432, Dako), FITC (11-4317-87, eBioscience), and Alexa Fluor 633 (A21052, Life Technologies)

\section{Transmission Electron Microscopy Imaging}

Fibroblasts were exposed to UVC irradiation $\left(50 \mathrm{~J} / \mathrm{m}^{2}\right)$ and incubated for $24 \mathrm{~h}$. After treatment cells were fixated $\mathrm{O} / \mathrm{N}$ with McDowells fixative; $1 \%$ glutaraldehyde, $4 \%$ paraformaldehyde in $0.1 \mathrm{M}$ phosphate buffer. After washing with phosphate buffer the specimens were postfixed with a solution of $1 \% \mathrm{OsO}_{4}$ in water. Subsequently, the specimens were dehydrated in an ethanol series and embedded in epoxy resin (LX112). For electron microscopic analysis ultrathin (70-nm) sections of the samples were cut with a diamond knife, collected on formvar-coated grids, and counterstained with uranyl acetate and lead citrate. All samples were examined and photographed in a FEI Tecnai T12 transmission electron microscope at the Electron Microscopy Centre Amsterdam.

\section{ADDITIONAL INFORMATION}

\section{Data Deposition and Access}

The PUS1 variant was deposited in ClinVar (https://www.ncbi.nlm.nih.gov/clinvar) under accession number SCV000995055.

\section{Ethics Statement}

Written informed consent was obtained from patients and/or parents prior to inclusion in this study, which was performed in accordance with the ethical standards of the Declaration of Helsinki. Informed consent was also obtained from four unrelated healthy control individuals. Approval for the study was obtained from the Medical Ethical Committee of the Academic Medical Center, University of Amsterdam, the Netherlands (2013_234/NL40331.078.12). 
Competing Interest Statement

The authors have declared no competing interest.

\section{Referees}

Thomas Liehr

Anonymous

Received May 23, 2019; accepted in revised form October 7, 2019.

\section{Acknowledgments}

Our most special thanks go to the family of the patient and to all healthy control volunteers who made this study possible. We also thank the electron microscopy experts Anita Grootemaat and Nicole van der Wel in the Cellular Imaging Department and the molecular DNA technicians in the Clinical Genetics Department at the Amsterdam UMC.

\section{Author Contributions}

A.W.M. and E.G.D. conceived of and designed the study. M.P. and T.W.K. treated the patient and provided clinical data. M.A. and P.L. collected and analyzed clinical and genetic data. E.G.D., M.L., and A.M. performed experiments and analyzed the data. A.W.M. and R.H.H. supervised the project and analyzed data. E.G.D. and A.W.M. wrote the manuscript in consultation with M.A. and P.L. All authors reviewed and edited the final manuscript.

\section{Funding}

R.H.H. is supported by a European Research Council (ERC) Starting grant (no. 638290), a Vidi grant from ZonMw (no. 91715305), and a grant from the Velux Stiftung (\#1063). A.W.M. is supported under the frame of E-Rare-2, ERA-Net for Research on Rare Diseases ZonMw \#40-44000-98-1008 in the Netherlands.

\section{REFERENCES}

Badhai J, Fröjmark AS, J. Davey E, Schuster J, Dahl N. 2009. Ribosomal protein S19 and S24 insufficiency cause distinct cell cycle defects in Diamond-Blackfan anemia. Biochim Biophys Acta 1792: 1036-1042. doi:10.1016/j.bbadis.2009.08.002

Balbeur S, Grisart B, Parmentier B, Sartenaer D, Leonard PE, Ullmann U, Boulanger S, Leroy L, Ngendahayo P, Lungu-Silviu $C$, et al. 2016. Trisomy rescue mechanism: the case of concomitant mosaic trisomy 14 and maternal uniparental disomy 14 in a 15-year-old girl. Clin Case Rep 4: 265-271. doi:10.1002/ccr3.501

Boczonadi V, Horvath R. 2014. Mitochondria: impaired mitochondrial translation in human disease. Int $J$ Biochem Cell Biol 48: 77-84. doi:10.1016/j.biocel.2013.12.011

Burrage LC, Tang S, Wang J, Donti TR, Walkiewicz M, Luchak JM, Chen LC, Schmitt ES, Niu Z, Erana R, et al. 2014. Mitochondrial myopathy, lactic acidosis, and sideroblastic anemia (MLASA) plus associated with a novel de novo mutation $(\mathrm{m} .8969 \mathrm{G}>\mathrm{A})$ in the mitochondrial encoded ATP6 gene. Mol Genet Metab 113: 207-212. doi:10.1016/j.ymgme.2014.06.004

Bykhovskaya Y, Casas K, Mengesha E, Inbal A, Fischel-Ghodsian N. 2004. Missense mutation in pseudouridine synthase 1 (PUS1) causes mitochondrial myopathy and sideroblastic anemia (MLASA). Am J Hum Genet 74: 1303-1308. doi:10.1086/421530

Campagnoli MF, Ramenghi U, Armiraglio M, Quarello P, Garelli E, Carando A, Avondo F, Pavesi E, Fribourg S, Gleizes PE, et al. 2008. RPS19 mutations in patients with Diamond-Blackfan anemia. Hum Mutat 29: $911-$ 920. doi:10.1002/humu.20752

Cao M, Donà M, Valentino ML, Valentino L, Semplicini C, Maresca A, Cassina M, Torraco A, Galletta E, Manfioli $V$, et al. 2016. Clinical and molecular study in a long-surviving patient with MLASA syndrome due to novel PUS1 mutations. Neurogenetics 17: 65-70. doi:10.1007/s10048-015-0465-x

Casas KA, Fischel-Ghodsian N. 2004. Mitochondrial myopathy and sideroblastic anemia. Am J Med Genet A 125A: 201-204. doi:10.1002/ajmg.a.20368

Danilova N, Sakamoto KM, Lin S. 2008. Ribosomal protein S19 deficiency in zebrafish leads to developmental abnormalities and defective erythropoiesis through activation of p53 protein family. Blood 112: 52285237. doi:10.1182/blood-2008-01-132290

Dutt S, Narla A, Lin K, Mullally A, Abayasekara N, Megerdichian C, Wilson FH, Currie T, Khanna-Gupta A, Berliner $N$, et al. 2011. Haploinsufficiency for ribosomal protein genes causes selective activation of p53 in human erythroid progenitor cells. Blood 117: 2567-2576. doi:10.1182/blood-2010-07-295238

el-Deiry WS, Tokino T, Velculescu VE, Levy DB, Parsons R, Trent JM, Lin D, Mercer WE, Kinzler KW, Vogelstein B. 1993. WAF1, a potential mediator of p53 tumor suppression. Cell 75: 817-825. doi:10.1016/0092-8674 (93)90500-P 
Fernandez-Vizarra E, Berardinelli A, Valente L, Tiranti V, Zeviani M. 2007. Nonsense mutation in pseudouridylate synthase 1 (PUS1) in two brothers affected by myopathy, lactic acidosis and sideroblastic anaemia (MLASA). J Med Genet 44: 173-180. doi:10.1136/jmg.2006.045252

Fleming MD. 2011. Congenital sideroblastic anemias: iron and heme lost in mitochondrial translation. Hematol Am Soc Hematol Educ Program 2011: 525-531. doi:10.1182/asheducation-2011.1.525

Fulda S, Galluzzi L, Kroemer G. 2010. Targeting mitochondria for cancer therapy. Nat Rev Drug Discov 9: 447464. doi:10.1038/nrd3137

Inbal A, Avissar N, Shaklai M, Kuritzky A, Schejter A, Ben-David E, Shanske S, Garty BZ. 1995. Myopathy, lactic acidosis, and sideroblastic anemia: a new syndrome. Am J Med Genet 55: 372-378. doi:10.1002/ajmg 1320550325

Kasapkara CS, Tümer L, Zanetti N, Ezgü F, Lamantea E, Zeviani M. 2017. A myopathy, lactic acidosis, sideroblastic anemia (MLASA) case due to a novel PUS1 mutation. Turk J Haemato/ 34: 376-377. doi:10.4274/tjh .2017 .0231

King DA, Fitzgerald TW, Miller R, Canham N, Clayton-Smith J, Johnson D, Mansour S, Stewart F, Vasudevan P, Hurles ME, et al. 2014. A novel method for detecting uniparental disomy from trio genotypes identifies a significant excess in children with developmental disorders. Genome Res 24: 673-687. doi:10.1101/gr .160465 .113

Latonen L, Taya Y, Laiho M. 2001. UV-radiation induces dose-dependent regulation of p53 response and modulates p53-HDM2 interaction in human fibroblasts. Oncogene 20: 6784-6793. doi:10.1038/sj.onc .1204883

Liehr T. 2019. Cases with uniparental disomy. http://upd-tl.com/upd.html (accessed 01/20/2019).

Liu CY, Lee CF, Hong CH, Wei YH. 2004. Mitochondrial DNA mutation and depletion increase the susceptibility of human cells to apoptosis. Ann N Y Acad Sci 1011: 133-145. doi:10.1196/annals.1293.014

Metodiev MD, Assouline Z, Landrieu P, Chretien D, Bader-Meunier B, Guitton C, Munnich A, Rötig A. 2015. Unusual clinical expression and long survival of a pseudouridylate synthase (PUS1) mutation into adulthood. Eur J Hum Genet 23: 880-882. doi:10.1038/ejhg.2014.192

Patton JR, Bykhovskaya Y, Mengesha E, Bertolotto C, Fischel-Ghodsian N. 2005. Mitochondrial myopathy and sideroblastic anemia (MLASA): missense mutation in the pseudouridine synthase 1 (PUS1) gene is associated with the loss of tRNA pseudouridylation. J Biol Chem 280: 19823-19828. doi:10.1074/jbc .M500216200

Pereboeva L, Westin E, Patel T, Flaniken I, Lamb L, Klingelhutz A, Goldman F. 2013. DNA damage responses and oxidative stress in dyskeratosis congenita. PLoS One 8: e76473. doi:10.1371/journal .pone.0076473

Perkins G, Bossy-Wetzel E, Ellisman MH. 2009. New insights into mitochondrial structure during cell death. Exp Neurol 218: 183-192. doi:10.1016/j.expneurol.2009.05.021

Raben N, Sherman JB. 1995. Mutations in muscle phosphofructokinase gene. Hum Mutat 6: 1-6. doi:10.1002/ humu.1380060102

Raisova M, Hossini AM, Eberle J, Riebeling C, Wieder T, Sturm I, Daniel PT, Orfanos CE, Geilen CC. 2001. The $\mathrm{Bax} / \mathrm{Bcl}$-2 ratio determines the susceptibility of human melanoma cells to CD95/Fas-mediated apoptosis. J Invest Dermatol 117: 333-340. doi:10.1046/j.0022-202x.2001.01409.x

Richardson DR, Lane DJ, Becker EM, Huang ML, Whitnall M, Suryo Rahmanto Y, Sheftel AD, Ponka P. 2010. Mitochondrial iron trafficking and the integration of iron metabolism between the mitochondrion and cytosol. Proc Natl Acad Sci 107: 10775-10782. doi:10.1073/pnas.0912925107

Riley LG, Cooper S, Hickey P, Rudinger-Thirion J, McKenzie M, Compton A, Lim SC, Thorburn D, Ryan MT, Giegé $R$, et al. 2010. Mutation of the mitochondrial tyrosyl-tRNA synthetase gene, YARS2, causes myopathy, lactic acidosis, and sideroblastic anemia-MLASA syndrome. Am J Hum Genet 87: 52-59. doi:10 .1016/j.ajhg.2010.06.001

Robinson WP. 2000. Mechanisms leading to uniparental disomy and their clinical consequences. Bioessays 22: 452-459. doi:10.1002/(SICl)1521-1878(200005)22:5<452::AID-BIES7>3.0.CO;2-K

Suzuki T, Nagao A, Suzuki T. 2011. Human mitochondrial tRNAs: biogenesis, function, structural aspects, and diseases. Annu Rev Genet 45: 299-329. doi:10.1146/annurev-genet-110410-132531

Tesarova M, Vondrackova A, Stufkova H, Veprekova L, Stranecky V, Berankova K, Hansikova H, Magner M, Galoova N, Honzik T, et al. 2019. Sideroblastic anemia associated with multisystem mitochondrial disorders. Pediatr Blood Cancer 66: e27591. doi:10.1002/pbc.27591

Ulirsch JC, Verboon JM, Kazerounian S, Guo MH, Yuan D, Ludwig LS, Handsaker RE, Abdulhay NJ, Fiorini C, Genovese G, et al. 2018. The genetic landscape of Diamond-Blackfan anemia. Am J Hum Genet 103: 930 947. doi:10.1016/j.ajhg.2018.10.027

Vaseva AV, Moll UM. 2009. The mitochondrial p53 pathway. Biochim Biophys Acta 1787: 414-420. doi:10 .1016/j.bbabio.2008.10.005

Vora S. 1983. Isozymes of human phosphofructokinase: biochemical and genetic aspects. Isozymes Curr Top Biol Med Res 11: 3-23. 
Vousden KH, Lane DP. 2007. p53 in health and disease. Nat Rev Mol Cell Biol 8: 275-283. doi:10.1038/ nrm2147

Yarham JW, Elson JL, Blakely EL, McFarland R, Taylor RW. 2010. Mitochondrial tRNA mutations and disease. Wiley Interdiscip Rev RNA 1: 304-324. doi:10.1002/wrna.27

Zeharia A, Fischel-Ghodsian N, Casas K, Bykhocskaya Y, Tamari H, Lev D, Mimouni M, Lerman-Sagie T. 2005. Mitochondrial myopathy, sideroblastic anemia, and lactic acidosis: an autosomal recessive syndrome in Persian Jews caused by a mutation in the PUS1 gene. J Child Neurol 20: 449-452. doi:10.1177/ 08830738050200051301

Zhou L, Chang DC. 2008. Dynamics and structure of the Bax-Bak complex responsible for releasing mitochondrial proteins during apoptosis. J Cell Sci 121: 2186-2196. doi:10.1242/jcs.024703

Zinkel S, Gross A, Yang E. 2006. BCL2 family in DNA damage and cell cycle control. Cell Death Differ 13: 13511359. doi:10.1038/sj.cdd. 4401987 


\section{COLD SPRING HARBOR Molecular Case Studies}

\section{A uniparental isodisomy event introducing homozygous pathogenic variants drives a multisystem metabolic disorder}

Eileen G. Daniels, Marielle Alders, Marco Lezzerini, et al.

Cold Spring Harb Mol Case Stud 2019, 5: a004457 originally published online October 25, 2019 Access the most recent version at doi: $10.1101 /$ mcs.a004457
Supplementary http://molecularcasestudies.cshlp.org/content/suppl/2019/11/07/mcs.a004457.D Material C1

References This article cites 38 articles, 8 of which can be accessed free at: http://molecularcasestudies.cshlp.org/content/5/6/a004457.full.html\#ref-list-1

License This article is distributed under the terms of the Creative Commons Attribution-NonCommercial License, which permits reuse and redistribution, except for commercial purposes, provided that the original author and source are credited.

Email Alerting Receive free email alerts when new articles cite this article - sign up in the box at the Service top right corner of the article or click here. 